\title{
Shape Operators and Structure Tensors of Real Hypersurfaces in Nonflat Quaternionic Space Forms
}

by

\author{
Sadahiro MAEDA and Toshiaki ADACHI
}

Presented by Bogdan BOJARSKI

Summary. We characterize curvature-adapted real hypersurfaces in nonflat quaternionic space forms in terms of their shape operators and structure tensors.

1. Introduction. In a nonflat quaternionic space form, which is either a quaternionic projective space or a quaternionic hyperbolic space, we have the following nice examples of homogeneous real hypersurfaces. In a quaternionic projective space $\mathbb{H} P^{n}(c)$ of quaternionic sectional curvature $c$, they are

(A) a tube of radius $r \in(0, \pi / \sqrt{c})$ around the canonically embedded totally geodesic $\mathbb{H} P^{m}(c)$ for some $m \in\{0, \ldots, n-2\}$,

(M) a tube of radius $r \in(0, \pi / 2 \sqrt{c})$ around the canonically embedded totally geodesic complex projective space $\mathbb{C} P^{n}(c)$,

and in a quaternionic hyperbolic space $\mathbb{H} H^{n}(c)$ of quaternionic sectional curvature $c$, they are

(A) a horosphere in $\mathbb{H} H^{n}(c)$ and a tube of some radius $r \in(0, \infty)$ around the canonically embedded totally geodesic $\mathbb{H} H^{m}(c)$ for some $m \in\{0, \ldots, n-1\}$,

2000 Mathematics Subject Classification: Primary 53B25; Secondary 53C40.

Key words and phrases: real hypersurfaces, curvature-adapted real hypersurfaces, quaternionic space forms, shape operators, structure tensors, quaternionic Kähler structures.

The first author partially supported by Grant-in-Aid for Scientific Research (C) (No. 14540080), Ministry of Education, Science, Sports and Culture, Japan.

The second author partially supported by Grant-in-Aid for Scientific Research (C) (No. 14540075), Ministry of Education, Science, Sports and Culture, Japan. 
(M) a tube of some radius $r \in(0, \infty)$ around the canonically embedded totally geodesic complex hyperbolic space $\mathbb{C} H^{n}(c)$.

We call these examples a hypersurface of type (A) and of type (M) in a nonflat quaternionic space form $M^{n}(c ; \mathbb{H})$ of quaternionic sectional curvature $c(\neq 0)$, respectively. In this note we study their shape operators and structure tensors induced from the quaternionic structure on $M^{n}(c ; \mathbb{H})$.

2. Curvature-adapted real hypersurfaces. In order to study real hypersurfaces of type (A) and (M), Berndt [B] introduced the notion of curvature-adapted hypersurfaces in a Riemannian manifold $\widetilde{M}$. A hypersurface $M$ of a Riemannian manifold $\widetilde{M}$ is called curvature-adapted if the normal Jacobi operator $K$ and the shape operator $A$ of $M$ with respect to a unit normal vector field $\mathcal{N}$ are simultaneously diagonalizable (i.e. $K \circ A=A \circ K$ ). Here the normal Jacobi operator $K: T M \rightarrow T M$ of $M$ with respect to $\mathcal{N}$ is defined by $K(\cdot)=\widetilde{R}(\cdot, \mathcal{N}) \mathcal{N}$, where $\widetilde{R}$ is the curvature tensor of $\widetilde{M}$. For a real hypersurface $M$ in a quaternionic Kähler manifold $\widetilde{M}$ with quaternionic Kähler structure $\mathcal{J}$, which is a rank 3 vector subbundle of the bundle of endomorphisms of the tangent bundle $T M$, we decompose $T M$ into $\mathcal{D} \oplus \mathcal{D}^{\perp}$, where $\mathcal{D}$ is the maximal subbundle of $T M$ which is invariant by $\mathcal{J}$. Here, a quaternionic Kähler structure $\mathcal{J}$ on a Riemannian manifold $\widetilde{M}$ of real dimension $4 n$ is a rank 3 vector subbundle of the bundle of endomorphisms of $T \widetilde{M}$ with the following properties:

1) For each point $\widetilde{x} \in \widetilde{M}$ there is an open neighborhood $\widetilde{G}$ of $\widetilde{x}$ in $\widetilde{M}$ and sections $J_{1}, J_{2}, J_{3}$ of the restriction $\left.\mathcal{J}\right|_{\widetilde{G}}$ over $\widetilde{G}$ such that

(i) each $J_{i}$ is an almost Hermitian structure on $\widetilde{G}$, that is, $J_{i}^{2}=-$ id and

$$
\left\langle J_{i} \tilde{X}, \tilde{Y}\right\rangle+\left\langle\widetilde{X}, J_{i} \tilde{Y}\right\rangle=0 \quad \text { for all vector fields } \widetilde{X} \text { and } \widetilde{Y} \text { on } \widetilde{G},
$$

where $\langle$,$\rangle is the Riemannian metric of \widetilde{M}$,

(ii) $J_{i} J_{i+1}=J_{i+2}=-J_{i+1} J_{i}(i \bmod 3)$ for $i=1,2,3$.

2) $\widetilde{\nabla}_{\widetilde{X}} J$ is a section of $\mathcal{J}$ for each vector field $\widetilde{X}$ on $\widetilde{M}$ and section $J$ of the bundle $\mathcal{J}$, where $\widetilde{\nabla}$ denotes the Riemannian connection of $\widetilde{M}$.

When the ambient space $\widetilde{M}$ is a nonflat quaternionic space form, curvatureadapted real hypersurfaces are characterized in terms of $\mathcal{D}$ and the shape operators: The following three conditions on a real hypersurface $M$ in $M^{n}(c ; \mathbb{H})$ are equivalent:

(1) $M$ is curvature-adapted.

(2) The subbundle $\mathcal{D}$ is invariant under the shape operator of $M$.

(3) The subbundle $\mathcal{D}^{\perp}$ is invariant under the shape operator of $M$. 
It was shown by Berndt $[\mathrm{B}]$ that every curvature-adapted real hypersurface in $\mathbb{H} P^{n}(c)$ is locally congruent to a hypersurface of type (A) or (M) and that every curvature-adapted real hypersurface in $\mathbb{H} H^{n}(c)$ all of whose principal curvatures are constant is locally congruent to a hypersurface of type (A) or $(\mathrm{M})$.

3. Structure tensors and the shape operator. Let $M$ be a real hypersurface in a quaternionic Kähler manifold $\widetilde{M}$. For an endomorphism $J \in \mathcal{J}$ we define the structure tensor $\phi_{J}: T M \rightarrow T M$ associated with $J$ by $\phi_{J}=\left.\pi \circ J\right|_{T M}$, where $\pi:\left.T \widetilde{M}\right|_{M} \rightarrow T M$ is the canonical projection. Let $\mathcal{S}=\left\{\phi_{J} \mid J \in \mathcal{J}\right\}$ be the set of all structure tensors. This is a rank 3 subbundle of the bundle of endomorphisms of $T M$. We set $\xi_{J}=-J \mathcal{N}$ for each $J \in \mathcal{J}$. It is clear that $\mathcal{D}_{x}^{\perp}=\left\{\xi_{J}(x) \mid J \in \mathcal{J}\right\}$ at each point $x \in M$ and that $\phi_{J}\left(\xi_{J}\right)=0, \phi_{J}\left(\mathcal{D}^{\perp}\right) \subset \mathcal{D}^{\perp}$ and $\phi_{J}(v)=J v$ for every $v \in \mathcal{D}$.

In a complex projective space, real hypersurfaces of type (A), which are tubes around canonically embedded totally geodesic complex projective spaces, are characterized as hypersurfaces with $A \phi=\phi A$. Here $\phi$ is the structure tensor induced by the complex structure of the ambient space.

We denote by $\mathfrak{F}(X)$ the set of real functions on a domain $X$. As in the case of complex space form, we consider an endomorphism $f \phi A+g A \phi$ of $T M$ for $f, g \in \mathfrak{F}(T M)$ and $\phi \in \mathcal{S}$, which is given by $(f \phi A+g A \phi)(v)=$ $f(v) \phi(A v)+g(v) A \phi(v)$ for $v \in T M$.

Proposition 1. Let $M$ be a real hypersurface of a nonflat quaternionic space form $\widetilde{M}^{n}(c ; \mathbb{H})$. Then the following conditions are equivalent:

(1) $M$ is curvature-adapted.

(2) For every $\phi \in \mathcal{S}$ there exists $f \in \mathfrak{F}(T M)$ satisfying $(f \phi A+A \phi)(\mathcal{D})$ $\subset \mathcal{D}$.

$\left(2^{\prime}\right)(f \phi A+g A \phi)(\mathcal{D}) \subset \mathcal{D}$ for every $\phi \in \mathcal{S}$ and $f, g \in \mathfrak{F}(T M)$.

(3) For every $\phi \in \mathcal{S}$ there exists $g \in \mathfrak{F}(T M)$ satisfying $(\phi A+g A \phi)\left(\mathcal{D}^{\perp}\right)$ $\subset \mathcal{D}^{\perp}$.

$\left(3^{\prime}\right)(f \phi A+g A \phi)\left(\mathcal{D}^{\perp}\right) \subset \mathcal{D}^{\perp}$ for every $\phi \in \mathcal{S}$ and $f, g \in \mathfrak{F}(T M)$.

Proof. $(1) \Rightarrow\left(2^{\prime}\right) \&\left(3^{\prime}\right)$. This is trivial since $A(\mathcal{D}) \subset \mathcal{D}$ and $A\left(\mathcal{D}^{\perp}\right) \subset \mathcal{D}^{\perp}$. $\left(2^{\prime}\right) \Rightarrow(2)$ and $\left(3^{\prime}\right) \Rightarrow(3)$ are trivial.

$(3) \Rightarrow(1)$. We decompose $A \xi_{J}$ as $A \xi_{J}=\widehat{\xi}_{J}+\xi_{J}^{\perp} \in \mathcal{D} \oplus \mathcal{D}^{\perp}$ for each $\xi_{J} \in \mathcal{D}^{\perp}$. We then have

$$
\mathcal{D}^{\perp} \ni\left(\phi_{J} A+g A \phi_{J}\right)\left(\xi_{J}\right)=\phi_{J} A \xi_{J}=\phi_{J}\left(\widehat{\xi}_{J}\right)+\phi_{J}\left(\xi_{J}^{\perp}\right) .
$$

As $\phi_{J}(\widehat{\xi}) \in \mathcal{D}$ and $\phi_{J}\left(\xi_{J}^{\perp}\right) \in \mathcal{D}^{\perp}$, this implies $\phi_{J}(\widehat{\xi})=0$, so that $\widehat{\xi}=0$. Thus we see that $A\left(\mathcal{D}^{\perp}\right) \subset \mathcal{D}^{\perp}$ and $M$ is curvature-adapted.

$(2) \Rightarrow(1)$. For each $x \in M$ we take a local basis $J_{1}, J_{2},\left.J_{3} \in \mathcal{J}\right|_{G}$ on a neighborhood of $x$ with $J_{i}^{2}=-1$ and $J_{i} \circ J_{i+1}=J_{i+2}=-J_{i+1} \circ J_{i}(i \bmod 3)$. 
Putting $\phi_{i}=\phi_{J_{i}}$ and $\xi_{i}=\xi_{J_{i}}$, we express $A v$ for each $v \in \mathcal{D}$ as

$$
A v=\widehat{v}+\eta_{1}(v) \xi_{1}+\eta_{2}(v) \xi_{2}+\eta_{3}(v) \xi_{3} \quad \text { with } \widehat{v} \in \mathcal{D} .
$$

Then by assumption we have

$$
\begin{aligned}
\mathcal{D} \ni & \left(f \phi_{1} A+A \phi_{1}\right)(v) \\
= & f(v)\left\{\phi_{1}(\widehat{v})+\eta_{2}(v) \xi_{3}-\eta_{3}(v) \xi_{2}\right\} \\
& +\left\{\widehat{\phi_{1}(v)}+\eta_{1}\left(\phi_{1}(v)\right) \xi_{1}+\eta_{2}\left(\phi_{1}(v)\right) \xi_{2}+\eta_{3}\left(\phi_{1}(v)\right) \xi_{3}\right\} .
\end{aligned}
$$

Hence $\eta_{1}\left(\phi_{1}(v)\right)=0$, and similarly $\eta_{2}\left(\phi_{2}(v)\right)=\eta_{3}\left(\phi_{3}(v)\right)=0$. Thus we can see that $\eta_{i}(v)=\eta_{i}\left(\phi_{i}\left(-\phi_{i}(v)\right)\right)=0$ for each $i=1,2,3$, so that $A(\mathcal{D}) \subset \mathcal{D}$. Therefore $M$ is curvature-adapted in $M^{n}(c ; \mathbb{H})$.

As a consequence of Proposition 1 we establish the following characterization of hypersurfaces of type (A) in $\mathbb{H} P^{n}(c)$.

THEOREM 1. The following conditions on a real hypersurface $M$ of $\mathbb{H} P^{n}(c)$ are equivalent:

(1) $M$ is of type (A).

(2) $\phi A=A \phi$ for each $\phi \in \mathcal{S}$.

(3) For each $\phi \in \mathcal{S}$ there exists $g \in \mathfrak{F}(T M)$ with $\phi A+g A \phi=0$ on $\mathcal{D}^{\perp}$.

(4) For each $\phi \in \mathcal{S}$ there exists $f \in \mathfrak{F}(T M)$ with $f \phi A+A \phi=0$ on $\mathcal{D}$.

Proof. Proposition 1 guarantees that $M$ is curvature-adapted in $\widetilde{M}^{n}(\mathbb{H} ; c)$, hence $M$ is either of type (A) or of type (M) under each condition. We denote by $\lambda_{j}$ an eigenvalue of $\left.A\right|_{\mathcal{D}}$ and by $\mu_{j}$ that of $\left.A\right|_{\mathcal{D}^{\perp}}$, and by $m(\nu)$ and $V_{\nu}$ the multiplicity and the eigenspace corresponding to the eigenvalue $\nu$, respectively. The following is due to Berndt [B]:

When $M$ is a hypersurface of type (A), its tangent bundle decomposes as $T M=V_{\lambda_{1}} \oplus V_{\lambda_{2}} \oplus V_{\mu_{1}}$ with

$$
\lambda_{1}=\frac{\sqrt{c}}{2} \cot \frac{\sqrt{c} r}{2}, \quad \lambda_{2}=-\frac{\sqrt{c}}{2} \tan \frac{\sqrt{c} r}{2}, \quad \mu_{1}=\sqrt{c} \cot (\sqrt{c} r),
$$

and each of the eigenspaces $V_{\lambda_{1}}, V_{\lambda_{2}}$ and $V_{\mu_{1}}$ is invariant under every $\phi \in \mathcal{S}$. (For the case when $M$ is a geodesic sphere, $V_{\lambda_{2}}=\{0\}$.) Therefore it is clear that $\phi A=A \phi$ for each $\phi \in \mathcal{S}$ in this case. When $M$ is of type (M), its tangent bundle decomposes as $T M=V_{\lambda_{1}} \oplus V_{\lambda_{2}} \oplus V_{\mu_{1}} \oplus V_{\mu_{2}}$, where

$$
\begin{array}{rlrl}
\lambda_{1} & =\frac{\sqrt{c}}{2} \cot \frac{\sqrt{c} r}{2}, & \lambda_{2}=-\frac{\sqrt{c}}{2} \tan \frac{\sqrt{c} r}{2}, \\
\mu_{1}=\sqrt{c} \cot (\sqrt{c} r), & \mu_{2}=-\sqrt{c} \tan (\sqrt{c} r),
\end{array}
$$

and $m\left(\mu_{1}\right)=1, m\left(\mu_{2}\right)=2$. For each point we can take a local basis $J_{i}, i=$ $1,2,3$, satisfying $J_{i}^{2}=-1, J_{i} \circ J_{i+1}=J_{i+2}=-J_{i+1} \circ J_{i}(i \bmod 3)$ and 


$$
\begin{aligned}
& \phi_{1}\left(V_{\lambda_{j}}\right)=V_{\lambda_{j}}(j=1,2), \quad \phi_{1}\left(V_{\mu_{1}}\right)=\{0\}, \quad \phi_{1}\left(V_{\mu_{2}}\right)=V_{\mu_{2}}, \\
& \phi_{i}\left(V_{\lambda_{1}}\right)=V_{\lambda_{2}}, \quad \phi_{i}\left(V_{\lambda_{2}}\right)=V_{\lambda_{1}}, \quad \phi_{i}\left(V_{\mu_{1}}\right) \subset V_{\mu_{2}}, \\
& \phi_{i}\left(V_{\mu_{2}}\right)=V_{\mu_{1}}(i=2,3),
\end{aligned}
$$

where $\phi_{i}=\phi_{J_{i}}$.

What we have to show is that condition (3) or (4) implies $M$ is of type (A).

$(3) \Rightarrow(1)$. For each $J \in \mathcal{J},(3)$ leads us to $\phi_{J}\left(A \xi_{J}\right)=-g(\xi) A \phi_{J}\left(\xi_{J}\right)=0$. Hence $A \xi_{J}$ is proportional to $\xi_{J}$, which shows $\xi_{J}$ is principal. As $D_{x}^{\perp}=$ $\left\{\xi_{J}(x) \mid J \in \mathcal{J}\right\}$ for each $x$, it should be an eigenspace of $\left.A\right|_{\mathcal{D}_{x}}$. Considering principal curvatures of real hypersurfaces of type (A) and of type (M), we find that $M$ is not of type (M). Every hypersurface of type (A) clearly satisfies (3) with $g \equiv-1$. Thus $M$ is of type (A).

$(4) \Rightarrow(1)$. By assumption, $A \phi v=-f(v) \phi A v$ for every $v \in \mathcal{D}$. When $M$ is of type (M), we consider a vector $v=a_{1} v_{1}+a_{2} v_{2} \in \mathcal{D}$ with $a_{1}, a_{2} \in \mathbb{R}$ and $v_{1} \in V_{\lambda_{1}}, v_{2} \in V_{\lambda_{2}}, v_{j} \neq 0$. Since $\lambda_{1} \neq \lambda_{2}$, we see that $A \phi_{i}(v)$ is not proportional to $\phi_{i} A v$ for the structure tensor $\phi_{i}, i=2,3$, associated with the local basis given above. When $M$ is of type (A), it satisfies (4) with $f \equiv-1$. Thus $M$ is of type (A).

Inspecting the proof of Proposition 1, we can improve the statement as follows:

Proposition 2. For a real hypersurface $M$ in a nonflat $M^{n}(c ; \mathbb{H})$, the following conditions are equivalent:

(1) $M$ is curvature-adapted in $\widetilde{M}^{n}(c ; \mathbb{H})$.

$\left(2^{\prime \prime}\right)$ For each $x \in M$ there exists a basis $\left\{K_{1}, K_{2}, K_{3}\right\}$ of $\mathcal{J}_{x}$ and functions $f_{1}, f_{2}, f_{3} \in \mathfrak{F}\left(T_{x} M\right)$ satisfying $\left(f_{i} \phi_{K_{i}} A+A \phi_{K_{i}}\right)\left(\mathcal{D}_{x}\right) \subset \mathcal{D}_{x}$ for $i=1,2,3$.

$\left(3^{\prime \prime}\right)$ For each $x \in M$ there exists a basis $\left\{K_{1}, K_{2}, K_{3}\right\}$ of $\mathcal{J}_{x}$ and functions $g_{1}, g_{2}, g_{3} \in \mathfrak{F}\left(T_{x} M\right)$ satisfying $\left(\phi_{K_{i}} A+g_{i} A \phi_{K_{i}}\right)\left(\mathcal{D}_{x}^{\perp}\right) \subset \mathcal{D}_{x}^{\perp}$ for $i=1,2,3$.

In this context we can improve Theorem 1 in the following manner.

THEOREM 2. For a real hypersurface $M$ in a quaternionic projective space $\mathbb{H} P^{n}(c)$ the following conditions are equivalent:

(1) $M$ is of type $(A)$.

$\left(2^{\prime}\right)$ For each $x \in M$ there exists a basis $\left\{K_{1}, K_{2}, K_{3}\right\}$ of $\mathcal{J}_{x}$ satisfying $\phi_{K_{i}} A=A \phi_{K_{i}}$ for $i=1,2,3$.

$\left(3^{\prime}\right)$ For each $x \in M$ there exists a basis $\left\{K_{1}, K_{2}, K_{3}\right\}$ of $\mathcal{J}_{x}$ and $g_{1}, g_{2}, g_{3}$ $\in \mathfrak{F}\left(T_{x} M\right)$ satisfying $\phi_{K_{i}} A+g_{i} A \phi_{K_{i}}=0$ on $\mathcal{D}_{x}^{\perp}$ for $i=1,2,3$.

$\left(4^{\prime}\right)$ For each $x \in M$ there exists a basis $\left\{K_{1}, K_{2}, K_{3}\right\}$ of $\mathcal{J}_{x}$ and $f_{1}, f_{2}, f_{3}$ $\in \mathfrak{F}\left(T_{x} M\right)$ satisfying $f_{i} \phi_{K_{i}} A+A \phi_{K_{i}}=0$ on $\mathcal{D}_{x}$ for $i=1,2,3$. 
Proof. Proposition 2 guarantees that under each condition, $M$ is curvature-adapted in $\mathbb{H} P^{n}(c)$, hence it is either of type (A) or of type (M). Reviewing the proof of Theorem 1 , we only need to check that $\left(4^{\prime}\right)$ implies (1). When $M$ is of type (M), we take a local basis $J_{i}, i=1,2,3$, of $\mathcal{J}$ satisfying $J_{i}^{2}=-1, J_{i} \circ J_{i+1}=J_{i+2}=-J_{i+1} \circ J_{i}(i \bmod 3)$ and (3.1). Setting $K_{i}=\sum_{j=1}^{3} a_{i j} J_{j}$ we may suppose $a_{33} \neq 0$. We then have

$$
\begin{aligned}
& A \phi_{K_{3}}\left(b_{1} \xi_{J_{1}}+b_{2} \xi_{J_{2}}\right)=-\mu_{1} a_{33} b_{2} \xi_{1}+\mu_{2} a_{33} b_{1} \xi_{2}+\mu_{2}\left(a_{31} b_{2}-a_{32} b_{1}\right) \xi_{3}, \\
& \phi_{K_{3}} A\left(b_{1} \xi_{J_{1}}+b_{2} \xi_{J_{2}}\right)=-\mu_{2} a_{33} b_{2} \xi_{1}+\mu_{1} a_{33} b_{1} \xi_{2}+\left(\mu_{2} a_{31} b_{2}-\mu_{1} a_{32} b_{1}\right) \xi_{3},
\end{aligned}
$$

for some constants $b_{1}, b_{2}$. Hence $A \phi_{K_{3}}\left(b_{1} \xi_{J_{1}}+b_{2} \xi_{J_{2}}\right)$ is not neccesarily proportional to $\phi_{K_{3}} A\left(b_{1} \xi_{J_{1}}+b_{2} \xi_{J_{2}}\right)$, and $M$ is not of type (M). When $M$ is of type (A), it clearly satisfies $\left(4^{\prime}\right)$.

In order to characterize homogeneous real hypersurfaces in a complex projective space $\mathbb{C} P^{n}$, Kimura $[\mathrm{K}]$ studied commutativity of two endomorphisms derived from the shape operators and structure tensors (see Proposition 3 below). Here, we also consider endomorphisms $P=P_{\phi, f}=\phi A+f A \phi$ and $Q=Q_{\phi, g, k}=\phi A+g A \phi+k \phi$ of $T M$ for functions $f, g, k: M \rightarrow \mathbb{R}$. When we consider $P, Q$ on a tangent space $T_{x} M$, we treat $f, g, k$ as constants.

LEMma. Let $M$ be a real hypersurface in a quaternionic Kähler manifold $\widetilde{M}$. If $\left(P_{\phi_{J}, f} Q_{\phi_{J}, g, k}-Q_{\phi_{J}, g, k} P_{\phi_{J}, f}\right) \xi_{J}(x)=0$ at some $x \in M$ with some constants $f, g, k$ with $f \neq g$, then $\xi_{J}(x)$ is a principal curvature vector of $M$ in $\widetilde{M}$.

Proof. Direct computation yields

$$
\begin{aligned}
& P_{\phi_{J}, f} Q_{\phi_{J}, g, k}-Q_{\phi_{J}, g, k} P_{\phi_{J}, f} \\
& =(f-g)\left(-\phi_{J} A^{2} \phi_{J}+A \phi_{J}^{2} A\right)+k\left\{(1-f) \phi_{J} A \phi_{J}+f A \phi_{J}^{2}-\phi_{J}^{2} A\right\},
\end{aligned}
$$

in particular,

$$
\begin{aligned}
& \left(P_{\phi_{J}, f} Q_{\phi_{J}, g, k}-Q_{\phi_{J}, g, k} P_{\phi_{J}, f}\right) \xi_{J}=(f-g) A \phi_{J}^{2} A \xi_{J}-k \phi_{J}^{2} A \xi_{J} \\
& \quad=(f-g) A\left(-A \xi_{J}+\frac{\left\langle A \xi_{J}, \xi_{J}\right\rangle}{\left\|\xi_{J}\right\|^{2}} \xi_{J}\right)-k\left(-A \xi_{J}+\frac{\left\langle A \xi_{J}, \xi_{J}\right\rangle}{\left\|\xi_{J}\right\|^{2}} \xi_{J}\right) .
\end{aligned}
$$

Hence

$$
\begin{aligned}
0 & =\left\langle\left(P_{\phi_{J}, f} Q_{\phi_{J}, g, k}-Q_{\phi_{J}, g, k} P_{\phi_{J}, f}\right) \xi_{J}(x), \xi_{J}(x)\right\rangle \\
& =(f(x)-g(x))\left(-\left\|A \xi_{J}\right\|^{2}+\frac{\left\langle A \xi_{J}(x), \xi_{J}(x)\right\rangle^{2}}{\left\|\xi_{J}\right\|^{2}}\right),
\end{aligned}
$$

which shows that $\left\|A \xi_{J}(x)\right\|^{2}=\left\langle A \xi_{J}(x), \xi_{J}(x)\right\rangle^{2} /\left\|\xi_{J}\right\|^{2}$. Thus we conclude that $\xi_{J}(x)$ is principal.

REMARK. On every hypersurface of type (A) in a nonflat $M^{n}(c ; \mathbb{H})$ the commutation relation $P Q=Q P$ holds for arbitrary $\phi \in \mathcal{S}$ and functions $f, g, k$ because $\phi A=A \phi$. 
In view of the Lemma we obtain the following:

TheOREM 3. For a real hypersurface $M$ in $\mathbb{H} P^{n}(c)$ the following conditions are equivalent:

(1) $M$ is of type (A).

(2) $P_{\phi, f} Q_{\phi, g, k}=Q_{\phi, g, k} P_{\phi, f}$ for all $\phi \in \mathcal{S}$ and $f, g, k \in \mathfrak{F}(M)$.

$\left(2^{\prime}\right)$ For each $\phi \in \mathcal{S}$ there exist $f, g, k \in \mathfrak{F}(M)$ such that $f-g$ has no zeros and $P_{\phi, f} Q_{\phi, g, k}=Q_{\phi, g, k} P_{\phi, f}$.

(3) $P_{\phi, f} Q_{\phi, g, k}=Q_{\phi, g, k} P_{\phi, f}$ on $\mathcal{D}^{\perp}$ for all $\phi \in \mathcal{S}$ and $f, g, k \in \mathfrak{F}(M)$.

$\left(3^{\prime}\right)$ For each $\phi \in \mathcal{S}$ there exist $f, g, k \in \mathfrak{F}(M)$ such that $f-g$ has no zeros and $P_{\phi, f} Q_{\phi, g, k}=Q_{\phi, g, k} P_{\phi, f}$ on $\mathcal{D}^{\perp}$.

(4) For each $x \in M$ there exists a basis $\left\{K_{1}, K_{2}, K_{3}\right\}$ of $\mathcal{J}_{x}$ and constants $f_{i}, g_{i}, k_{i}$ such that $f_{i} \neq g_{i}$ and

$$
P_{\phi_{K_{i}}, f_{i}} Q_{\phi_{K_{i}}, g_{i}, k_{i}}=Q_{\phi_{K_{i}}, g_{i}, k_{i}} P_{\phi_{K_{i}}, f_{i}} \quad \text { on } T_{x} M \text { for } i=1,2,3 \text {. }
$$

Proof. Under each condition it follows from the Lemma that $A \mathcal{D}^{\perp} \subset$ $\mathcal{D}^{\perp}$, so that our real hypersurface $M$ is curvature-adapted. When $M$ is of type (A), these conditions trivially hold. Therefore we assume that $M$ is of type (M). Since there is a non-principal vector in $\mathcal{D}^{\perp}$, condition $\left(3^{\prime}\right)$ does not hold. Suppose $M$ satisfies (4). Then we may consider $\xi_{K_{1}} \in V_{\mu_{1}}$ and $\xi_{K_{2}}, \xi_{K_{3}} \in V_{\mu_{2}}$ by the Lemma. Since $\left\{K_{1}, K_{2}, K_{3}\right\}$ is a basis of $\mathcal{J}_{x}$, we see that $\phi_{K_{2}}\left(\xi_{K_{3}}\right)=a \xi_{K_{1}}$ with a nonzero constant $a$ and $\phi_{K_{2}}\left(\xi_{K_{1}}\right) \in V_{\mu_{2}} \backslash\{0\}$. As $\phi_{K_{2}}\left(V_{\lambda_{1}}\right)=V_{\lambda_{2}}, \phi_{K_{2}}\left(V_{\lambda_{2}}\right)=V_{\lambda_{1}}$, for $v \in V_{\lambda_{1}}$ we find by (3.2) that

$$
\begin{gathered}
\left(P_{\phi_{K_{2}}, f_{2}} Q_{\phi_{K_{2}}, g_{2}, k_{2}}-Q_{\phi_{K_{2}}, g_{2}, k_{2}} P_{\phi_{K_{2}}, f_{2}}\right) v \\
\quad=\left(\lambda_{2}-\lambda_{1}\right)\left\{\left(f_{2}-g_{2}\right)\left(\lambda_{1}+\lambda_{2}\right)+k_{2}\left(f_{2}-1\right)\right\} v, \\
\left(P_{\phi_{K_{2}}, f_{2}} Q_{\phi_{K_{2}}, g_{2}, k_{2}}-Q_{\phi_{K_{2}}, g_{2}, k_{2}} P_{\phi_{K_{2}}, f_{2}}\right) \xi_{K_{3}} \\
=a\left(\mu_{2}-\mu_{1}\right)\left\{\left(f_{2}-g_{2}\right)\left(\mu_{1}+\mu_{2}\right)+k_{2}\left(f_{2}-1\right)\right\} \phi_{K_{2}}\left(\xi_{K_{1}}\right) .
\end{gathered}
$$

Since $\lambda_{1}+\lambda_{2} \neq \mu_{1}+\mu_{2}$, this is a contradiction which proves our result.

In terms of $\mathcal{D}^{\perp}$, we have the following characterization of all curvatureadapted real hypersurfaces of $\mathbb{H} P^{n}(c)$ :

THEOREm 4. For a real hypersurface $M$ in $\mathbb{H} P^{n}(c)$ the following conditions are equivalent:

(1) $M$ is curvature-adapted.

(2) For each $x \in M$ there exists a basis $\left\{K_{1}, K_{2}, K_{3}\right\}$ of $\mathcal{J}_{x}$ and constants $f_{i}, g_{i}, k_{i}$ such that $f_{i} \neq g_{i}$ and

$$
P_{\phi_{K_{i}}, f_{i}} Q_{\phi_{K_{i}}, g_{i}, k_{i}}=Q_{\phi_{K_{i}}, g_{i}, k_{i}} P_{\phi_{K_{i}}, f_{i}} \quad \text { on } \mathcal{D}_{x}^{\perp} \text { for } i=1,2,3 \text {. }
$$

(3) There exist constants $f, g, k(f \neq g)$ such that for each $x$ we can choose a basis $\left\{K_{1}, K_{2}, K_{3}\right\}$ of $\mathcal{J}_{x}$ satisfying

$$
P_{\phi_{K_{i}}, f} Q_{\phi_{K_{i}}, g, k}=Q_{\phi_{K_{i}}, g, k} P_{\phi_{K_{i}}, f} \quad \text { on } \mathcal{D}_{x}^{\perp} \text { for } i=1,2,3 \text {. }
$$


Proof. By the Lemma, (2) implies $A \mathcal{D}^{\perp} \subset \mathcal{D}^{\perp}$, hence $M$ is curvatureadapted. On the other hand, when $M$ is of type (M), we take $f=-1, g=1$ and $k=-\left(\mu_{1}+\mu_{2}\right)$. For a local basis $J_{i}, i=1,2,3$, of $\mathcal{J}$ satisfying $J_{i}^{2}=-1$, $J_{i} \circ J_{i+1}=J_{i+2}=-J_{i+1} \circ J_{i}(i \bmod 3)$ and $(3.1)$, we see that $P_{\phi_{J_{i}}, f}=0$ on $V_{\mu_{1}}$ and $Q_{\phi_{J_{i}}, g, k}=0$ on $V_{\mu_{2}}$. Hence (3) holds. Thus we obtain the result.

We end this paper with some results corresponding to Theorems 3 and 4 on real hypersurfaces in a nonflat complex space form $M^{n}(c ; \mathbb{C})$ of constant holomorphic sectional curvature $c(\neq 0)$, which is either a complex projective space or a complex hyperbolic space. We say that a real hypersurface $M$ in $M^{n}(c ; \mathbb{C})$ is a Hopf hypersurface if the characteristic vector $\xi$ of $M$ is principal.

Proposition 3. For a real hypersurface $M$ in a nonflat complex space form $M^{n}(c ; \mathbb{C})$, two endomorphisms $P=\phi A-A \phi$ and $Q_{k}=\phi A+A \phi+k \phi$ commute for some constant $k$ if and only if $M$ is locally congruent to a Hopf hypersurface all of whose principal curvatures are constant.

Proof. For $c>0$, the statement was proved by Kimura $[\mathrm{K}]$.

As we have

$$
P Q_{k}-Q_{k} P=2 \phi A^{2} \phi-2 A \phi^{2} A+k\left(2 \phi A \phi-A \phi^{2}-\phi^{2} A\right)
$$

and $\phi^{2} v=-v+\langle v, \xi\rangle \xi$ for an arbitrary tangent vector $v$, we see that

$$
\begin{aligned}
\left\langle\left(P Q_{k}-Q_{k} P\right) \xi, \xi\right\rangle & =\left\langle 2 A^{2} \xi-\langle A \xi, \xi\rangle A \xi+k(A \xi-\langle A \xi, \xi\rangle \xi), \xi\right\rangle \\
& =2\|A \xi\|^{2}-2\langle A \xi, \xi\rangle^{2} .
\end{aligned}
$$

Thus if $P Q_{k}-Q_{k} P=0$ we find that $\xi$ is principal, so that the corresponding principal curvature $\alpha$ is constant (see $[\mathrm{NR}]$ ).

Let $v$ be a principal vector orthogonal to $\xi$. If $A v=\lambda v$, then we have $2(2 \lambda-\alpha) A \phi v=(2 \alpha \lambda+c) \phi v$. We first consider the case $2 \lambda \neq \alpha$. Then $\phi v$ is also a principal vector of principal curvature $(2 \alpha \lambda+c) /\{2(2 \lambda-\alpha)\}$ (see $[\mathrm{NR}])$. By (3.3) we have

$$
\left(\lambda-\frac{2 \alpha \lambda+c}{2(2 \lambda-\alpha)}\right)\left(\lambda+\frac{2 \alpha \lambda+c}{2(2 \lambda-\alpha)}+k\right)=0,
$$

hence either $4 \lambda^{2}-4 \alpha \lambda+c=0$ or $4 \lambda^{2}+4 k \lambda-2 k \alpha+c=0$. Therefore in this case each principal curvature function is locally constant on $M$. Next we study the case that there is a point such that $\lambda=\alpha / 2$ is a principal curvature. By continuity of principal curvature functions the above argument guarantees that $\alpha / 2$ is a principal curvature on some neighborhood of this point. So our real hypersurface $M$ is locally congruent to a Hopf hypersurface with constant principal curvatures.

We now check that every Hopf hypersurface with constant principal curvatures satisfies $P Q_{k}=Q_{k} P$ for some constant $k$. Such real hypersurfaces 
are classified completely. In a complex hyperbolic space $\mathbb{C} H^{n}(c)$ they are called real hypersurfaces of type (A) and (B) (for details, see [NR]). For a real hypersurface of type $(\mathrm{A})$, which is either a horosphere or a tube of radius $r(0<r<\infty)$ around a totally geodesic $\mathbb{C} H^{d}(c)$ with $0 \leq d \leq n-1$, as we have $P=\phi A-A \phi=0$, the claim is obvious. For a real hypersurface $M$ of type (B), which is a tube of radius $r$ around a totally geodesic real hyperbolic space $\mathbb{R} H^{n}(c / 4)$ of constant sectional curvature $c / 4$, the tangent bundle decomposes as $T M=V_{\lambda_{1}} \oplus V_{\lambda_{2}} \oplus \mathbb{R} \xi$, where

$\lambda_{1}=\frac{\sqrt{|c|}}{2} \operatorname{coth} \frac{\sqrt{|c|} r}{2}, \quad \lambda_{2}=\frac{\sqrt{|c|}}{2} \tanh \frac{\sqrt{|c|} r}{2}, \quad \alpha=\sqrt{|c|} \tanh (\sqrt{|c|} r)$, and $\phi\left(V_{\lambda_{1}}\right)=V_{\lambda_{2}}, \phi\left(V_{\lambda_{2}}\right)=V_{\lambda_{1}}$. Therefore $Q_{k}=0$ with $k=-\left(\lambda_{1}+\lambda_{2}\right)=$ $-\sqrt{|c|} \operatorname{coth}(\sqrt{|c|} r)$, hence $P Q_{k}=Q_{k} P$.

In a complex projective space $\mathbb{C} P^{n}(c)$ Hopf hypersurfaces with constant principal curvatures are real hypersurfaces of types $(\mathrm{A})-(\mathrm{E})$ (see $[\mathrm{NR}])$. For a real hypersurface of type (A), which is a tube of radius $r(<\pi / \sqrt{c})$ around a totally geodesic $\mathbb{C} P^{d}(c)$ with $1 \leq d \leq n-1$, the statement is obvious as $P=0$. For a real hypersurface $M$ of type (B), which is a tube of radius $r(<\pi /(2 \sqrt{c}))$ around a totally geodesic real projective space $\mathbb{R} P^{n}(c / 4)$ of constant sectional curvature $c / 4$, the tangent bundle decomposes as $T M=$ $V_{\lambda_{1}} \oplus V_{\lambda_{2}} \oplus \mathbb{R} \xi$, where

$$
\lambda_{1}=-\frac{\sqrt{c}}{2} \cot \frac{\sqrt{c} r}{2}, \quad \lambda_{2}=\frac{\sqrt{c}}{2} \tan \frac{\sqrt{c} r}{2}, \quad \alpha=\sqrt{c} \tan (\sqrt{c} r),
$$

and $\phi\left(V_{\lambda_{1}}\right)=V_{\lambda_{2}}, \phi\left(V_{\lambda_{2}}\right)=V_{\lambda_{1}}$. Therefore $Q_{k}=0$ with $k=-\left(\lambda_{1}+\lambda_{2}\right)=$ $\sqrt{c} \cot (\sqrt{|c|} r)$, hence $P Q_{k}=Q_{k} P$. For a real hypersurface $M$ of type (C), (D) or $(\mathrm{E})$, which is a tube of radius $r(<\pi /(2 \sqrt{c}))$ around $\mathbb{C} P^{1}(c) \times$ $\mathbb{C} P^{(n-1) / 2}(c)$, complex Grassmannian $\mathbb{C} G_{2,5}$ or $S O(10) / U(5)$, respectively, the tangent bundle decomposes as $T M=V_{\lambda_{1}} \oplus V_{\lambda_{2}} \oplus V_{\lambda_{3}} \oplus V_{\lambda_{4}} \oplus \mathbb{R} \xi$, where

$$
\begin{aligned}
& \lambda_{1}=\frac{\sqrt{c}}{2} \cot \frac{\sqrt{c} r}{2}, \quad \lambda_{2}=-\frac{\sqrt{c}}{2} \tan \frac{\sqrt{c} r}{2}, \quad \lambda_{3}=\frac{\sqrt{c}(1+\tan (\sqrt{c} r / 2))}{2(1-\tan (\sqrt{c} r / 2))}, \\
& \lambda_{4}=-\frac{\sqrt{c}(1-\tan (\sqrt{c} r / 2))}{2(1+\tan (\sqrt{c} r / 2))}, \quad \alpha=\sqrt{c} \cot (\sqrt{c} r),
\end{aligned}
$$

$\phi\left(V_{\lambda_{i}}\right)=V_{\lambda_{i}}, i=1,2$, and $\phi\left(V_{\lambda_{3}}\right)=V_{\lambda_{4}}, \phi\left(V_{\lambda_{4}}\right)=V_{\lambda_{3}}$. We consider $Q_{k}$ for $k=-\left(\lambda_{3}+\lambda_{4}\right)=\sqrt{c} \tan \sqrt{c} r$. Since

$$
\begin{aligned}
& P\left(V_{\lambda_{i}}\right)=0, \quad Q_{k}\left(V_{\lambda_{i}}\right) \subset V_{\lambda_{i}}(i=1,2), \\
& Q_{k}\left(V_{\lambda_{j}}\right)=0(j=3,4), \quad P\left(V_{\lambda_{3}}\right) \subset V_{\lambda_{4}}, \quad P\left(V_{\lambda_{4}}\right) \subset V_{\lambda_{3}},
\end{aligned}
$$

we find $P Q_{k}=Q_{k} P=0$ and obtain our result.

Remark. In Proposition 3 we cannot relax the condition on $k$. Even in a complex projective space there exist Hopf hypersurfaces satisfying $P Q_{k}=$ 
$Q_{k} P$ for some function $k$ and having some principal curvatures not constant (see $[\mathrm{K}]$ for details).

\section{References}

[AM] T. Adachi and S. Maeda, Curvature-adapted real hypersurfaces in quaternionic space forms, Kodai Math. J. 24 (2001), 98-119.

[B] J. B. Berndt, Real hypersurfaces in quaternionic space forms, J. Reine Angew. Math. 419 (1991), 9-26.

[K] M. Kimura, Some non-homogeneous real hypersurfaces in a complex projective space I (Construction), II (Characterization), Bull. Fac. Education Ibaraki Univ. (Natural Sci.) 44 (1995), 1-16 and 17-31.

[NR] R. Niebergall and P. J. Ryan, Real hypersurfaces in complex space forms, in: Tight and Taut Submanifolds, T. E. Cecil and S. S. Chern (eds.), Cambridge Univ. Press, 1998, 233-305.

Sadahiro Maeda

Department of Mathematics

Shimane University

Matsue, Shimane, 690-8504, Japan

E-mail: smaeda@math.shimane-u.ac.jp
Toshiaki Adachi

Department of Mathematics Nagoya Institute of Technology Gokiso, Nagoya, 466-8555, Japan

E-mail: adachi@nitech.ac.jp

Received February 6, 2004;

received in final form March 23, 2004 